\title{
Behavioral resilience in the post-genomic era: emerging models linking genes with environment
}

\author{
Richard Rende* \\ Department of Psychiatry and Human Behavior, Transdisciplinary Research Group, Butler Hospital and Alpert Medical School of Brown University, \\ Providence, RI, USA
}

Edited by:

João R. M. Oliveira, Federal

University of Pernambuco, Brazil

\section{Reviewed by:}

Rebecca Elliott, University of

Manchester, UK

João R. M. Oliveira, Federal

University of Pernambuco, Brazil

Jonas Hannestad, Yale School of

Medicine, USA

${ }^{*}$ Correspondence:

Richard Rende, Department of Psychiatry and Human Behavior,

Transdisciplinary Research Group, Butler Hospital, Alpert Medical

School of Brown University, 345

Blackstone Blvd., Providence 02906

RI, USA.

e-mail: richardrendephd@gmail.com
One of the most important deliverables of the post-genomic era has been a new and nuanced appreciation of how the environment shapes-and holds potential to alter-the expression of susceptibility genes for behavioral dimensions and disorders. This paper will consider three themes that have emerged from cutting-edge research studies that utilize newer molecular genetic approaches as well as tried-and-true genetic epidemiological methodologies, with particular reference to evolving perspectives on resilience and plasticity. These themes are: (1) evidence for replicable and robust shared environmental effects on a number of clinically relevant behaviors in childhood and adolescence; (2) evolving research on gene-environment interaction; and (3) a newer focus on differential susceptibility and plasticity. The net sum of these themes is that consideration of genetic effects on behavioral dimensions and disorders needs to be connected to thinking about the role of environment as a potent source for promoting resilience and change.

Keywords: behavioral resilience, genes, shared environment, gene-environment interaction, differential susceptibility

\section{INTRODUCTION}

Over the past decade, genetic research on human behaviorboth normative behaviors and clinically relevant dimensions and disorders- has reached new levels of intensity. The results of genetically informative studies are now commonplace in mainstream journals in psychology and psychiatry and no longer the province of just specialty journals. As the focus on genetics has spread, and more interdisciplinary researchers have become part of the collective voice, a number of new models are emerging that provide conceptual guideposts to the varied ways that genes influence development-and the multiple factors that can influence gene expression.

Perhaps the most fundamental perspective to emerge in the post-genomic era is that gene expression with relevance for behavior occurs within salient and powerful environmental contexts. This statement goes way beyond outdated and non-specific debates about nature and nurture. Rather, both classical behavioral genetic approaches (which rely on statistical partitioning of genetic and environmental effects on individual differences in dimensions or disorders) and newer paradigms that incorporate specific gene markers have led to refinements of prior conceptual models as well as the generation of alternative frameworks. As much of the work to date has focused on developmental issues, I will make particular (but not exclusive) reference to studies on children and adolescents.

In this paper I will highlight three themes that are making important inroads in our understanding of how genetic differences amongst individuals leads to meaningful differences in behavioral development. In particular, I am coming from the perspective of what "resilience" may mean in the post-genomic era. As an overall statement, what we will consider is the extent to which "risk genes" lead to adverse behavioral outcomes from the perspective of gene-environment interplay. The general model which holds for nearly every behavioral dimension or disorder is the classic multifactorial framework which posits that a number of genetic loci and a number of environmental (or more specifically non-genetic) factors come together to shape the expression of phenotypes. The key issue now in the post-genomic era is to consider a number of potential models in which these two streams of influence-genetic and environmental-intersect to either express the consequences of risk or provide some buffer against adverse developmental outcomes. Thus, the three themes presented here will be discussed from the lens of a risk-resilience perspective.

\section{THEME ONE: THERE ARE SYSTEMATIC ENVIRONMENTAL INFLUENCES ON BEHAVIORAL DIMENSIONS AND DISORDERS IN ADDITION TO GENETIC EFFECTS}

For decades, most behavioral genetic research-natural experiments such as the classic twin design-suggested that individual differences in nearly all behavioral phenotypes were the product of two primary sources of influence-genes and non-shared environment. The bottom line of this type of quantitative model was that genes were the driving force behind both familial similarity and dissimilarity, as there was no evidence for systematic effects of the environment that would promote similarity between family members. From the perspective of resilience, then, there would be two primary processes to consider for a given behavioral disorder. 
First, family members (for example, siblings) would of course both share, and not share, risk genes to varying degrees- thus the luck of the DNA draw would put siblings at higher versus lower risk and reflect the extent to which resilience would even be a relevant construct (kids at low genetic risk have no need to be resilient from the genomic perspective). Second, there would be unique environmental influences-unique in the sense that they do not contribute to familial similarity and reflect individual-specific environmental factors-that would impact siblings differently and could represent potential pathways to resilience. It is noted that part of the non-shared environmental effect would arise from genetic differences. For example, youth with (theoretically speaking) higher doses of risk genes could react differently to the environment or be exposed to particular environmental events because of their genetic make-up. Resilience in this model could come from unique environmental effects that provide some type of buffer against the expression of risk genes.

While this model is plausible, a newer perspective is emerging from a number of genetically informative studies which suggests that shared environmental factors-non-genetic influences which promote similarity between family members-may in fact exert potent effects on risk for a number of behavioral dimensions and disorders in childhood and adolescence. Take, for example, the etiology of substance use in adolescence, the typical period of first onset. It is certainly the case that the initiation of substance use (e.g., tobacco, alcohol, marijuana), and quantitative estimates of amount of use, in adolescence has been shown to reflect heritable influences. However, many reviews have concluded that shared environmental factors also play a critical role in the initiation/use of substances in adolescence and some behavioral genetics see this as the dominant force which shapes precocious exposure to substances (see Rende and Waldman, 2006; Rende et al., 2009; Rende, 2011). As these reviews have suggested, this is a consistent finding across many genetically informative studies and across multiple cultures. It is also becoming apparent that shared environmental effects can not only have immediate effects but in fact become more influential during the transition from adolescence to early adulthood (Distel et al., 2011). Furthermore, there is evidence that they may be especially important at the extremes of behavior - for example, shared environmental effects are differentially more influential on high-levels of adolescent smoking than on the full range of individual differences (Rende et al., 2005).

It is worth emphasizing that this pattern of results has not only been found for adolescent substance use. There has been a similar recognition of, and appreciation for, the role of shared environment as a significant predictor of delinquent/antisocial behavior in early adolescence and the stability of these high-risk behaviors throughout adolescence (Burt et al., 2007, 2010, 2011). In addition, a number of studies have shown high-levels of depressive symptoms in adolescence are unduly influenced by shared environmental effects, whereas the broad range of individual differences does not show such evidence (Rende and Waldman, 2006).

One of the key aspects of this work on shared environment is that systematic effects have been replicated. The non-shared environmental model emphasizes individual-specific environmental effects. While these are undoubtedly important for individuals, the strong evidence for shared environmental effects suggests more lawful associations between environment and behavioral outcome, particularly for clinically salient phenotypes such as substance use and delinquent behavior. Recent studies are beginning to uncover moderators of these shared environmental effects. For example, we have utilized a powerful twin-sibling design-via analysis of the genetically informative subsample of the National Longitudinal Study of Adolescent Developmentalong with a measure of the sibling relationship to examine if sibling dynamics explain some of the shared environmental effect on adolescent substance use. Our work (Rende et al., 2005; Slomkowski et al., 2005) has suggested a sibling contagion model: having a connected relationship-including spending time together, liking each other, and having mutual friendshipswith a sibling who uses substances is not only highly predictive of use, it functions as a strong shared environmental effect (after controlling for genetic relatedness). Other genetically informative studies confirm that shared environmental influences-rather than genetic relatedness-represent the most salient pathways to sibling similarity for substance use in adolescence (Samek and Rueter, 2011). Family process researchers have emphasized the important role that siblings play in creating family-wide dynamics that promote risk for behavioral problems (Reiss et al., 2000; Rende et al., 2005, 2009; Slomkowski et al., 2001, 2005, 2009). Using data from the genetically informative NEAD project, Reiss et al. (2000) have provided extensive evidence that parental and sibling interactions are empirically linked via environmental processes (even after controlling for genetic similarity), forming a socially driven family sub-system with predictive value for adolescent adjustment. Recent genetically informative studies (e.g., Burt et al., 2007) that utilize videotaped interaction data examining antisocial behavior provide an opportunity to control for confounds such as rater effects that may skew findings based on self-report data. In this work core parenting and sibling interactive behaviors have been shown to reflect shared environmental processes.

What are the implications here in terms of resilience in the face of a high genetic load for behavioral problems? A first primary one is that the robust and replicable shared environmental effects demonstrate convincingly that genes are not the only systematic streams of influence on behavioral development particularly prior to adulthood - and as a corollary not the only pathway for familial resemblance. While genetic risk is important, it is most likely expressed in the context of strong environmental forces that either promote or buffer risk-and may in fact be a constant influence on behavioral development throughout childhood and (at a minimum) through adolescence. So one consideration is that we must not overstate the importance of genes on behavioral development. They are a critical part of the equation, but not the only significant term. Given the intense interest in genomics these days, it is easy to lose sight that the sum of genetic risk for many behavioral conditions in youth is not only not deterministic, but probably of lesser magnitude than many would assume-especially if environmental measures are brought to bear on the issue.

From a more clinical perspective, many children and adolescents are at high-risk for a number of behavioral problems because of their environment (keep in mind that "shared 
environment" does not equate "family environment"-there could of course be systematic effects of local environment such as neighborhood effects). Given this, a primary way of promoting resilience given the (hypothetical) presence of "high-risk" genes would be to alter these systematic environmental effects to lower youth's risk threshold (one could refer here to the fundamental liability model that has been in play for over four decades). The basic lesson here is that if genetic effects are not deterministicand in some cases are of a lower magnitude than the effects attributable to the environment - then changing the environment may be sufficient to reduce risk for a number of common behavioral problems. And the more distal implication is that there is much plasticity around the effects of genes, and pathways to resilience do not inherently need to be biological in origin.

\section{THEME TWO: THERE IS A GROWING LITERATURE DOCUMENTING INTERACTION BETWEEN GENETIC MARKERS AND ENVIRONMENTAL INFLUENCES}

Theme one makes the point that, for behavioral dimensions and disorders, genes are simply part of the mix, and that environmental effects can be as influential and systematic. Notice, however, that genetic risk was discussed in a general way with no reference to actual genes. Over the last decade, highly influential work has been conducted which has taken this on-and shown repeatedly that risk alleles are differentially expressed based on the presence or absence of environmental risk.

This line of work was initiated nearly a decade ago by the publication of two highly influential papers showing evidence of gene-environment interaction-one on risk for conduct disorder (Caspi et al., 2002) and one on risk for depression (Caspi et al., 2003). In both cases specific risk alleles-drawn from prior biological research on candidate genes-were shown to predict high-levels of problem behavior, but only when crossed with specific environmental risk factors. This work led to many studies conducted over the past decade on gene-environment interaction.

Caspi et al. (2010) have provided a cogent review of studies deriving from their initial discovery that variation in the promoter region of the serotonin transporter gene (5-HTT/SLC6A4) moderated the link between stressful life events and depression. What's especially illuminating when thinking about resilience is that they cast this work as examples of genetic sensitivity to the environment. The idea here is that risk alleles may carry risk for behavioral disorders because they moderate reactions to the environment. The implication, then, is that specific gene markers are influential in combination with environmental triggers, not as main effects on behavioral dimensions and disorders.

A recent paper illustrates this principle well. Uher et al. (2011) aimed this GxE framework at a critical clinical issue-predicting persistent depression versus single-episode depression. Seminal family studies conducted decades ago established convincingly that persistent depression (in those studies typically represented as early onset recurrent depression) aggregates strongly in families, whereas single-episode depression does not. The Uher et al. (2011) study offers a modern take on this by demonstrating (in two independent samples) that genetic risk-the presence of two short 5-HTTLPR alleles-confers an elevated risk for persistent (but not single-episode) depression when crossed with prior exposure to child maltreatment in childhood.

What does the conceptual and empirical work on GxE interaction imply about resilience? One proposition is that resilience is a complex process by which the adverse, genetically moderated reactions to environmental triggers are buffered through some other factor. In the case of depression, one could think of cognitive-behavioral therapies that help individuals learning coping mechanisms in the face of life stressors. Similarly, antidepressants may be operating by reversing the genetically moderated reactivity in the serotonin system to substantial stressors. The key conceptual point here is that, in the case of behavioral dimensions and disorders, risk alleles are not necessarily simply randomly activated, nor are they deterministically expressedrather they may primarily function by influencing the sensitivity to risk environments, consistent with the arguments put forth by Caspi et al. (2010). So resilience-and models that would generate ways of promoting resilience for those at higher genetic risk-becomes a more fine-grained proposition, and one with some potential as more studies establish strong linkages between specific risk alleles and known environmental triggers. The bottom line, though, is that resilience may in some cases involve changing the environment (the biological as well as psychosocial environment) in order to lower the impact of high-risk alleles.

\section{THEME THREE: GENES MAY CONVENE HIGH REACTIVITY TO BOTH GOOD AND BAD ENVIRONMENTAL INFLUENCES}

The GxE interaction model described above has historical roots in diathesis-stress models of psychopathology. Diathesis-stress models propose that clinically relevant phenotypes are the product of both high-risk genes and high-risk environments-risk genes and risk environments are each typically necessary but not sufficient to lead to disorder. The key breakthrough of course was to document this empirically and generate conceptual and methodological frameworks for examining GxE interaction in a forward-looking and informative way (Caspi et al., 2010). Over the past few years, though, this model has been tweaked in an interesting way with particular relevance for the concept of resilience-with the claim being that risk genes may also confer a particular ability to respond well to the environment.

The key idea is that specific "risk" genes (and relatedendophenotypic and phenotypic attributes and traits) do not always confer just vulnerability in the face of environmental adversity - rather they may also impart hyper-reactivity to positive experiences and influences and hence "differential susceptibility" to the environment (Belsky and Pluess, 2009). Of particular importance to this review is that Belsky and Pluess suggest that specific genes may function more like "plasticity factors" rather than "vulnerability factors." The overall suggestion is that genes may make some people much more reactive to the environment, and others much less so-and this would apply to both positive and negative environments.

Some recent examples will help illustrate the concept. Hankin and colleagues (2011) examined interactions between 
youths' 5-HTTLPR genotype (the gene referenced above in the work on depression) and degree of positive and supportive parenting (ranging from low to high) in three independent studies using a range of methods (including coded laboratory observations of child-parent interactions and parental and youth self-report). All three studies showed the same effect-youth homozygous for the functional short allele of 5-HTTLPR were more responsive to parenting, whether it was positive or negative. Youth at high genetic risk (the S'S' group) had low-levels of positive affect if their parents were unsupportive, but highlevels of positive affect if their parents were supportive. The key thing is that the S'S' group was most responsive to parentingand their responsiveness followed the emotional polarity of parenting. Thus, the S'S' group was not just vulnerable to negative parenting - they also profited the most from positive parenting.

Other papers have presented similar findings from a number of studies. There is, for example, evidence of differential susceptibility to the environment in youth as mediated by dopamine-related genes (dopamine receptor D2, DRD4, dopamine transporter). Bakermans-Kranenburg and van Ijzendoom (2011) report that children with "less efficient dopamine-related gene polymorphisms" had worse outcomes when they were in negative environments, but also showed the most benefit from being in positive environments. A similar conclusion was reached in a recent paper that focused on both serotonergic and dopaminergic genes, social environments, and aggression in youthkids with "reactive" genes either showed the highest or lowest levels of aggression depending on the valence of their social environment.

It is important to recognize that the findings to date have been reported for behavioral conditions such as aggression and emotional expression. It could be argued, of course, that such genetically moderated plasticity in response to different environments may not be the case for other behavioral disorders such as autism or dyslexia (it is, of course, an open question). Thus, it will be a conceptual and empirical task to examine how the principle of differential susceptibility will apply to different genes and gene systems, different sources of environmental influence, and different clinical phenotypes. That said, the differential susceptibility theory provides a novel model for how genes can reflect plasticity rather than risk for behavioral phenotypes-particularly as the key factor that shapes the translation from genotype to adaptive versus maladaptive outcome is the nature of the environment.

\section{REFERENCES}

Bakermans-Kranenburg, M. J., and van Ijzendoom, M. H. (2011). Differential susceptibility to rearing environment depending on dopamine-related genes: new evidence and a meta-analysis. Dev. Psychopathol. 23, 39-52.

Belsky, J., and Pluess, M. (2009). Beyond diathesis stress: differential susceptibility to environmental influences. Psychol. Bull. 135, 885-908.

Burt, S., Klahr, M., McGue, M., and Iacono, W. (2011). Confirming the etiology of adolescent actingout behaviors: an examination of observer-ratings in a sample of adoptive and biological siblings. J. Child Psychol. Psychiatry 52, 519-526.

Burt, S., McGue, M., and Iacono, W. (2010). Environmental con-

\section{CONCLUSIONS}

This brief overview highlights key conceptual and empirical advances in research at the intersection of genes and environment. Important themes from the perspective of resilience are that:

(1) New evidence for the importance of shared environment highlights that genetic influences are not the only systematic factors that result in familial similarity for a number of clinically relevant behaviors in childhood and adolescence. This implies that "risk" genes may not be as powerful as thought and they operate in the context of highly significant environmental currents. Thus, resilience with respect to risk genes can come from buffering provided by alternative environments which lower overall risk for disorder;

(2) Seminal work on GxE interaction-conducted nearly a decade ago-continues to resonate for a number of dimensions and disorders in childhood, adolescence, and adulthood. The body of evidence suggests that risk-promoting alleles are expressed and predict maladaptive functioning when crossed with environmental pathogens. As such, reductions in these pathogens could represent important pathways to resilience by turning off the triggers that activate riskpromoting alleles;

(3) Expansion of the GxE perspective includes considerations of differential susceptibility - the idea that a given "risk" allele may in fact signal high reactivity to the environment and phenotypes that match the valence of the environment (positive breeds positive; negative breeds negative). The important takeaway here is that, for some disorders, influential alleles may represent propensity for biological plasticity (the tendency to be strongly shaped by the environment) rather than risk or protection per se.

As genomic research on dimensions and disorders in childhood and adolescence continues at a rapid pace, there will undoubtedly be many more informative empirical papers that will refine the three themes reviewed in this article. Especially important will be understanding how each of these three themes may apply (more or less) for a range of behavioral outcomes. The overall implication, however, is clear-genes for behavior not only operate in a rich environmental context, but their expression is shaped in a dynamic interplay with the environment that can be observed empirically (Rende, 2011). As such, the concept of resilience-and the related-construct of plasticity-will continue to be expanded and refined.

tributions to the stability of antisocial behavior over time: are they shared or non-shared? J. Abnorm. Child Psychol. 38, 327-337.

Burt, S., McGue, M., Krueger, R., and Iacono, W. (2007). Environmental contributions to adolescent delinquency: a fresh look at the shared environment. J. Abnorm. Child Psychol. 35, 787-800.
Caspi, A., Hariri, A. R., Holmes, A., Uher, R., and Moffitt, T. E. (2010). Genetic sensitivity to the environment: the case of the serotonin transporter gene and its implications for studying complex diseases and traits. Am. J. Psychiatry 167, 509-527.

Caspi, A., McClay, J., Moffitt, T. E., Mill, J., Martin, J., Craig, I. W., Taylor, A., and Poulton, R. (2002). Role of 
genotype in the cycle of violence in maltreated children. Science 297 851-854.

Caspi, A., Sugden, K., Moffitt, T. E., Taylor, A., Craig, I. W., Harrington, H., McClay, J., Mill, J., Martin, J., Braithwaite, A., and Poulton, R. (2003). Influence of life stress on depression: moderation by a polymorphism in the 5-HTT gene. Science 301, 386-389.

Distel, M. A., Vink, J. M., Bartels, M., van Beijsterveldt, C. E., Neale, M. C., and Boomsma, D. I. (2011). Age moderates non-genetic influences on the initiation of cannabis use: a twin-sibling study in Dutch adolescents and young adults. Addiction 106, 1658-1666.

Hankin, B. L., Nederhof, E., Oppenheimer, C. W., Jenness, J., Young, J. F., Abela, J. R. Z., Smolen, A., Ormel, J., and Oldehinkel, A. J. (2011). Differential susceptibility in youth: evidence that 5-HTTLPR $\mathrm{X}$ positive parenting is associated with positive affect "for better and worse". Transl. Psychiatry 1, e44.

Reiss, D., Neiderhiser, J., Hetherington, E. M., and Plomin, R. (2000). The Relationship Code: Deciphering Genetic and Social Influences on
Adolescent Development. Cambridge, MA: Harvard University Press.

Rende, R. (2011). Interaction of genes and environment in adolescent substance use: opportunities for more tailored interventions. Mind and Brain, J. Psychiatry 2, 50-55.

Rende, R., Conti, D., Gilman, S., and Slomkowski, C. (2009). "Incorporating the social context in genetic studies of nicotine dependence," in National Cancer Institute Tobacco Control Monograph 22: Phenotypes and Endophenotypes: Foundations for Genetic Studies of Nicotine Use and Dependence. (Bethesda, MD: US Department of Health and Human Services). National Institutes of Health, National Cancer Institute. NIH Publication No. 09-6366, August 2009.

Rende, R., Slomkowski, C., Richardson, E., and Niaura, R. (2005). Sibling effects on substance use in adolescence: social contagion and genetic relatedness. J. Fam. Psychol. 19, 611-618.

Rende, R., and Waldman, I. (2006). "Behavioral and molecular genetics and developmental psychopathology," in Developmental
Psychopathology (Developmental Neuroscience, Vol. 2), eds D. Cicchetti and D. Cohen (New York, NY: Wiley and Sons), 427-464.

Samek, D. R., and Rueter, M. A. (2011). Considerations of elder sibling closeness in predicting younger sibling substance use: social learning versus social bonding. J. Fam. Psychol. 25, 931-941.

Slomkowski, C., Conger, K. J., Rende, R., Heylen, E., Little, W. M. Shebloski, B., Fox, P., Craine, J. L., and Conger, R. D. (2009). Sibling contagion for drinking in adolescence: a micro process framework. Eur. J. Dev. Sci. 3, 161-174.

Slomkowski, C., Rende, R., Conger, K. J., Simons, R., and Conger, R. D. (2001). Brothers, sisters, and delinquency: evaluating social influence from early to middle adolescence. Child Dev. 72, 271-283.

Slomkowski, C., Rende, R., Novak, S., Richardson, E., and Niaura, R. (2005). Sibling effects on smoking in adolescence: evidence for social influence from a geneticallyinformative design. Addiction 100, 430-438.

Uher, R., Caspi, A., Houts, R., Sugden, K., Williams, B., Poulton, R., and
Moffitt, T. E, (2011). Serotonin transporter gene moderates childhood maltreatment's effects on persistent but not single-episode depression: replications and implications for resolving inconsistent results. J. Affect. Disord. 135, 56-65.

Conflict of Interest Statement: The author declares that the research was conducted in the absence of any commercial or financial relationships that could be construed as a potential conflict of interest.

Received: 28 January 2012; accepted: 24 February 2012; published online: 22 March 2012.

Citation: Rende $R$ (2012) Behavioral resilience in the post-genomic era: emerg ing models linking genes with environment. Front. Hum. Neurosci. 6:50. doi: 10.3389/fnhum.2012.00050

Copyright (C) 2012 Rende. This is an open-access article distributed under the terms of the Creative Commons Attribution Non Commercial License, which permits non-commercial use, distribution, and reproduction in other forums, provided the original authors and source are credited. 